Ter ahertz t i me- domai $n$ spect roscopy of sul fur-cont ai ni ng bi orml ecul es

\begin{tabular}{|l|l|}
\hline 著者 & $\begin{array}{l}\text { YAMAMOTO Kohj i , KABI R MAl. Humay un, TOM NAGA } \\
\text { Kei suke }\end{array}$ \\
\hline $\begin{array}{l}\text { j our nal or } \\
\text { publ i cat i on t i t l e }\end{array}$ & Journal of the Opt i cal Soci ety of Aner i ca B \\
\hline vol une & 22 \\
\hline number & 11 \\
\hline page r ange & $2417-2426$ \\
\hline year & $2005-11$ \\
\hline URL & ht t p: //hdl . handl e. net /10098/3039 \\
\hline
\end{tabular}




\title{
Terahertz time-domain spectroscopy of sulfur-containing biomolecules
}

\author{
Kohji Yamamoto \\ Molecular Photoscience Research Center, Kobe University, Nada, Kobe 657-8501, Japan
}

Md. Humayun Kabir

Core Research for Evolutional Science and Technology, Japan Science and Technology Agency, Kobe University, Nada, Kobe 657-8501, Japan

Keisuke Tominaga

Molecular Photoscience Research Center, and Core Research for Evolutional Science and Technology, Japan Science and Technology Agency, Kobe University, Nada, Kobe 657-8501, Japan

Received January 3, 2005; revised manuscript received May 27, 2005

We present frequency-dependent absorption coefficients and refractive indices of sulfur-containing biomolecules in the far-infrared region measured by terahertz (THz) time-domain spectroscopy (TDS). The THz radiation was generated by a surface photocurrent method with a (100)InAs wafer as a $\mathrm{THz}$ emitter, and its intensity was enhanced by applying a magnetic field of $1.6 \mathrm{~T}$. The $\mathrm{THz}$ radiation was detected by the electrooptic sampling method with a ZnTe crystal. The characterization of the apparatus of THz TDS is discussed in detail. The spectral features in the $\mathrm{THz}$ region are different among sulfur-containing biomolecules of L,Lcystine, L-cysteine, L-methionine, and oxidized glutathione in powder. These results demonstrate that $\mathrm{THz}$ TDS is a promising method for studies on the low-frequency spectra of complex molecules and identification of materials. We present a calculation method to analyze the THz spectra based on a model function for dynamics of the dipoles, which allows us to obtain spectral information characterizing the low-frequency responses of the molecules. The experimental results are compared with ab initio calculations to discuss assignment of the vibrational modes. (C) 2005 Optical Society of America

OCIS codes: $300.6270,020.3690,320.7120$.

\section{INTRODUCTION}

There has been dramatic progress in the generation and detection techniques of freely propagating terahertz $(\mathrm{THz})$ radiation in the past two decades. The examples of the generation technique include photoconductive switching, ${ }^{1,2}$ optical rectification, ${ }^{3-5}$ and the surface photocurrent of semiconductors. ${ }^{6,7}$ Because the pulse duration of the $\mathrm{THz}$ radiation is in a subpicosecond time region, it is possible to measure the electric field of the radiation by coherent detection methods, which consequently allows us to conduct $\mathrm{THz}$ time-domain spectroscopy (TDS). By THz TDS we can obtain the refractive index and extinction coefficient of a medium by measuring the phase and amplitude of the radiation. $\mathrm{THz}$ TDS is an attractive method for studying dynamics in condensed phases with time scales of subpicoseconds and picoseconds. THz TDS has been widely applied to investigate various kinds of condensed materials, including neat liquids and mixtures of liquids, ${ }^{8-18}$ electrolyte solutions, ${ }^{19,20}$ biological polymers, ${ }^{21-23}$ biological molecules, ${ }^{24-28}$ supercritical fluid, ${ }^{29}$ and micelles. ${ }^{30-32}$

$\mathrm{THz}$ spectra obtained by $\mathrm{THz}$ TDS have the following relation with a power spectrum of a time correlation function (TCF) of a total dipole moment $\mathbf{M}(t)$ of the system $^{33-35}$ :

$$
\begin{aligned}
n(\widetilde{\nu}) \alpha(\widetilde{\nu}) \propto & \widetilde{\nu}[1-\exp (-\beta h c \widetilde{\nu})] \int_{-\infty}^{\infty} \mathrm{d} t \exp (-i 2 \pi c \tilde{\nu} t) \\
& \times\langle\mathbf{M}(0) \mathbf{M}(t)\rangle,
\end{aligned}
$$

where $n$ is the refractive index, $\alpha$ is the absorption coefficient, $\widetilde{\nu}$ is the wavenumber, and $\beta$ is $1 / k_{B} T . k_{B}, h, c$, and $T$ have the usual meanings. The TCF of $\mathbf{M}(t)$ carries information on dynamics such as low-frequency intermolecular and intramolecular vibrations, orientational relaxation of the dipoles, fluctuation of induced-dipole moments, and so on. These low-frequency modes are often affected by weak interactions of noncovalent bonds such as hydrogen bonding, charge-transfer interaction, and van der Waals forces. These interactions play crucial roles in chemical reactions, protein functions, and biological activities; therefore investigations of molecular dynamics and interactions in the low-frequency region provide fundamental information for the mechanisms of these processes.

In this paper we have constructed a THz TDS apparatus to measure frequency-dependent extinction coefficients and refractive indices of sulfur-containing biomolecules including amino acids (L,L-cystine, L-cysteine, L-methionine, and oxidized glutathione). We have devel- 


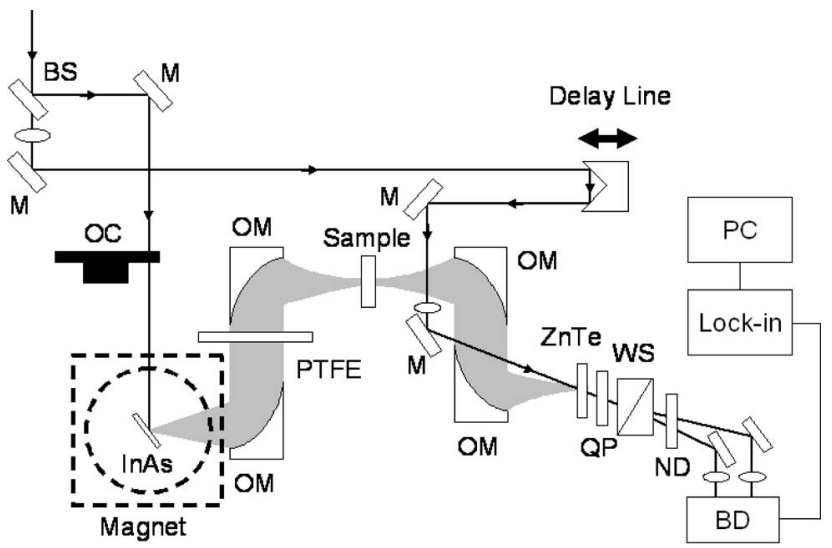

Fig. 1. Schematic diagram of our $\mathrm{THz}$ time-domain spectrometer. Pulsed THz radiation is generated from a (100)InAs surface and is detected by the EO-sampling technique using a (110)ZnTe crystal. A pair of permanent magnets provides a magnetic-flux density of $1.6 \mathrm{~T}$ in its center where the InAs plate is placed. A direction of the magnetic-flux density is parallel to the InAs surface. The plus sign of the magnetic-flux densities $\mathbf{B}$ is defined as the direction from top to bottom and the minus sign of $\mathbf{B}$ as the direction from bottom to top. InAs, $0.5 \mathrm{~mm}$ thick bulk (100)InAs with a single-sided polished surface; BS, beam splitter (transmittance is $10 \%$, reflectance is $90 \%$ ) for an optical pulse centered at $800 \mathrm{~nm}$; OC, optical chopper; OM, off-axis parabolic mirror (112.6 $\mathrm{mm}$ in effective focal length and $25.4 \mathrm{~mm}$ in diameter); ZnTe, 1 $\mathrm{mm}$ thick (110)ZnTe; QP, $\lambda / 4$ wave plate for optical pulses at 800 nm; WS, Wollaston prism; ND, neutral-density filter; BD, balanced silicon detector; PTFE, $3 \mathrm{~mm}$ thick polytetrafluoroethylene plate.

oped a procedure to analyze the spectra on the basis of a model function for the TCF of the total dipole moment. The low-frequency spectra of amino acids provide information on intermolecular interactions and conformational fluctuations, which are fundamental quantities to understand the formation of three-dimensional structures and biological functions of proteins and polypeptides. The low-frequency spectra of several amino acids have been investigated by $\mathrm{THz} \mathrm{TDS}^{26,27}$ and $a b$ initio calculations. ${ }^{36-40}$ We chose sulfur-containing amino acids for investigation, because the thiol group is highly polarizable and contributes to the stabilization of protein structure. The thiol group is also important because of its ability to form disulfide linkages that play a role in determining protein tertiary structure.

This paper is organized as follows. First, we briefly describe our THz TDS system that uses a (100)InAs wafer as a THz emitter and a (110)ZnTe crystal as an electrooptic (EO) detector. We next present a procedure to analyze $\mathrm{THz}$ spectra using a frequency-dependent complex permittivity based on the damping harmonic-oscillator model. The experimental results of the $\mathrm{THz}$ spectra are shown for sulfur biomolecules in powder, and the spectra are analyzed in terms of the theoretical model. Finally, we present results of $a b$ initio calculations of the biomolecules to discuss assignment of the vibrational modes.

\section{A. Experiment}

The THz TDS apparatus of generation and detection of the pulsed $\mathrm{THz}$ radiation is schematically depicted in Fig. 1. Femtosecond pulses at $800 \mathrm{~nm}$ with a pulse width of
$100 \mathrm{fs}$ are delivered from a mode-locked titanium:sapphire laser at a repetition rate of $82 \mathrm{MHz}$. The output power used for THz TDS is $500 \mathrm{~mW}$ on average. The femtosecond pulses are split into two parts with a ratio of 9:1 in power. Nine tenths of the pulse are used to generate the $\mathrm{THz}$ radiation, and the one tenth is used to detect the radiation. The pump pulses are focused onto a nondoped bulk (100)InAs surface with an incident angle of $45^{\circ}$. The (100)InAs plate with a thickness of $500 \mu \mathrm{m}$ and a singlesided polished surface is an $n$-type semiconductor with a carrier density of $\sim 3 \times 10^{16} \mathrm{~cm}^{-3}$. A pair of permanent magnets (Sumitsu \& Co.) provides a magnetic-flux density of $1.6 \mathrm{~T}$ in its center where the InAs plate is placed. A direction of the magnetic-flux density is parallel to the InAs surface. The $\mathrm{THz}$ radiation generated from the InAs surface is collected by an off-axis parabolic mirror. After reflection by the other three off-axis parabolic mirrors, the $\mathrm{THz}$ radiation is focused onto a (110)ZnTe crystal with a thickness of $1 \mathrm{~mm}$. All the off-axis parabolic mirrors with gold coating are $25.4 \mathrm{~mm}$ in diameter and $101.6 \mathrm{~mm}$ in effective focal length. The pump pulse reflected at the InAs surface is blocked by a polytetrafluoroethylene $\left[-\left(\mathrm{CF}_{2} \mathrm{CF}_{2}\right) n^{-}\right]$plate that is $3 \mathrm{~mm}$ thick. In the THz TDS measurements, samples are placed at the focused point of the radiation and oriented with its surface normal parallel to the radiation direction. The probe pulse passes through a ZnTe crystal, a $\lambda / 4$ wave plate, and a Wollaston prism. The prism separates the two components with different polarizations of the probe pulse, and the difference in intensity of the two components is detected by a balanced photodiode detector (Model 2007, New Focus) as a
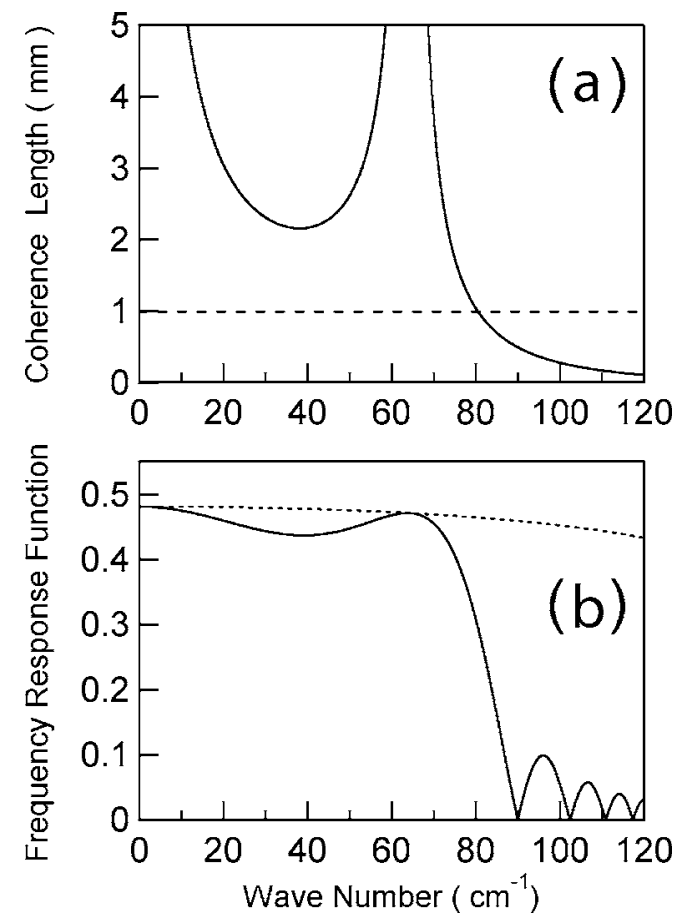

Fig. 2. EO-sampling efficiency of a $1 \mathrm{~mm}$ thick (110) $\mathrm{ZnTe}$ pumped by an optical pulse centered at $800 \mathrm{~nm}$ : (a) coherence length function, (b) frequency response function. Dotted curve in (b) depicts the real Fresnel coefficient, representing surface loss due to reflection. 
function of the delay time between the pump and the probe pulses. The signal is processed by a lock-in amplifier with a modulation frequency of $3.9 \mathrm{kHz}$.

Samples L,L-cystine, L-cysteine, L-methionine, and oxidized glutathione were purchased from Wako Pure Chemicals and were used without further purification. Pellets of these samples are prepared by an oil press without adding any substances, and the pressure was approximately $200 \mathrm{MPa}$. A sample thickness ranges from $100 \mu \mathrm{m}$ to $1 \mathrm{~mm}$, and a typical thickness is $300 \mu \mathrm{m}$. All the measurements were conducted at room temperature $\left(20^{\circ} \mathrm{C}\right)$.

\section{B. Data Analysis of Terahertz Time-Domain Spectroscopy}

In the normal incidence of the $\mathrm{THz}$ radiation to a sample in the plate, the ratio of complex electric fields of $\mathrm{THz}$ waves with and without transmission through the sample are expressed by ${ }^{41}$

$$
\frac{E_{S}(\widetilde{\nu})}{E_{R}(\widetilde{\nu})}=\frac{\frac{4 \widetilde{n}_{1}(\widetilde{\nu}) \tilde{n}_{2}(\widetilde{\nu})}{\left[\tilde{n}_{1}(\widetilde{\nu})+\tilde{n}_{2}(\widetilde{\nu})\right]^{2}} \exp \left\{i 2 \pi \widetilde{\nu}\left[n_{2}(\widetilde{\nu})-n_{1}(\widetilde{\nu})\right] d\right\} \exp \left\{-2 \pi \widetilde{\nu}\left[\left(\kappa_{2}(\widetilde{\nu})-\kappa_{1}(\widetilde{\nu})\right) d\right]\right\}}{1-\left[\frac{\tilde{n}_{1}(\widetilde{\nu})-\tilde{n}_{2}(\widetilde{\nu})}{\tilde{n}_{1}(\widetilde{\nu})+\tilde{n}_{2}(\widetilde{\nu})}\right]^{2} \exp \left[i 4 \pi \widetilde{\nu} n_{2}(\widetilde{\nu}) d\right] \exp \left[-4 \pi \widetilde{\nu} \kappa_{2}(\widetilde{\nu}) d\right]},
$$

where $E_{R}(\widetilde{\nu})$ and $E_{S}(\widetilde{\nu})$ are the reference and sample electric fields, respectively, and $d$ is the thickness of the sample. Subscripts 1 and 2 indicate parameters of air and the sample, respectively. $\widetilde{n}_{l}(\widetilde{\nu})$ is the complex refractive index represented by $\widetilde{n}_{l}(\widetilde{\nu})=n_{l}(\widetilde{\nu})+i \kappa_{l}(\widetilde{\nu})(l=1,2)$, where $n_{l}(\widetilde{\nu})$ and $\kappa_{l}(\widetilde{\nu})$ are the refractive index and the extinction coefficient, respectively. The denominator in Eq. (2) results from the multiple reflection within the sample, the socalled the etalon effect. The THz TDS analysis is conducted using Eq. (2) to extract $n_{2}(\widetilde{\nu})$ and $k_{2}(\widetilde{\nu})$ where $n_{1}(\widetilde{\nu})=1$ and $k_{1}(\widetilde{\nu})=0$ for the parameters of air. The absorption coefficient is obtained by $\alpha(\widetilde{\nu})=4 \pi \widetilde{\nu} \kappa(\widetilde{\nu}) / \ln 10$, represented by the logarithm with a base of 10 in this paper instead of a base of $e$ (Napier number). The dielectric permittivity $\left(\epsilon=\epsilon^{\prime}+i \epsilon^{\prime \prime}\right)$ is calculated from the relations $\epsilon^{\prime}=n^{2}-\kappa^{2}$ and $\epsilon^{\prime \prime}=2 n \kappa$.

\section{Method of Spectral Analysis}

Here we present a method of spectral analysis in the $\mathrm{THz}$ region by assuming a model function for the TCF of the total dipole moment $\mathbf{M}(t)$. According to the fluctuationdissipation theorem, an imaginary part of permittivity is related to the TCF of $\mathbf{M}(t)$ in the following way ${ }^{33-35}$ :

$$
\epsilon^{\prime \prime}(\widetilde{\nu})=\frac{1}{3} \frac{\pi}{\epsilon_{0} h c V}[1-\exp (-\beta h c \widetilde{\nu})] I_{M M}(\widetilde{\nu}),
$$

where

$$
\begin{gathered}
I_{M M}(\widetilde{\nu})=c \int_{-\infty}^{\infty} \mathrm{d} t \mathrm{C}_{M M}(t) \exp (-i 2 \pi c \tilde{\nu} t), \\
C_{M M}(t)=\langle\mathbf{M}(t) \mathbf{M}(0)\rangle .
\end{gathered}
$$

Here $\epsilon_{0}$ is the vacuum permittivity and $V$ is the volume of the system. $C_{M M}(t)$ is the TCF of $\mathbf{M}(t)$, and $I_{M M}(\widetilde{\nu})$ is the line-shape function. A prefactor of one third in Eq. (3a) results from the fact that the samples are randomly oriented, and this factor should be modified in the case of polarized samples. Both the real and the imaginary components of the permittivity are connected by the
Kramers-Kronig (K-K) relations. ${ }^{42}$ According to the K-K relation, the real component of the permittivity is calculated from its imaginary one:

$$
\epsilon^{\prime}(\widetilde{\nu})=\epsilon_{\infty}+\frac{2}{\pi} \int_{0}^{\infty} \mathrm{d} \widetilde{\nu}^{\prime} \epsilon^{\prime \prime}\left(\widetilde{\nu}^{\prime}\right) \frac{\widetilde{\nu}^{\prime}}{\widetilde{\nu}^{\prime 2}-\widetilde{\nu}^{2}} .
$$

We here assume that $\mathbf{M}(t)$ is expressed as a sum of individual dipole moments $\boldsymbol{\mu}_{k}(t), \mathbf{M}(t)=\Sigma_{k} \boldsymbol{\mu}_{k}(t)$. We further assume that the dipole moments do not correlate with each other and the TCF of $\mathbf{M}(t)$ can be expressed as a sum of the TCF of individual dipole moments $\left\langle\boldsymbol{\mu}_{k}(t) \boldsymbol{\mu}_{k}(0)\right\rangle$, $\langle\mathbf{M}(t) \mathbf{M}(0)\rangle=\Sigma\left\langle\boldsymbol{\mu}_{k}(t) \boldsymbol{\mu}_{k}(0)\right\rangle$

To proceed further, we adopt a model function for the TCF of $\mathbf{M}(t)$. In the simulation we try to reproduce the experimentally obtained real and imaginary components of the permittivity. We assume that the pure vibrational dephasing and lifetime of the excited state contribute to the broadening of the spectrum. One may also think that, in addition to these mechanisms of the line broadening, the reorientational relaxation of the dipole is important. However, since the sample is in a solid state in the present case, the contribution of the reorientational relaxation can be negligible. All these broadening mechanisms are represented into a single time constant $\tau_{j}$, where $j$ denotes the $j$ th vibrational mode, which we call a damping time constant. Within this damping harmonic-oscillator model, the TCF of the single dipole moment can be expressed as

$$
C_{\mu \mu}(t)=\left\langle\mu_{j}(t) \mu_{j}(0)\right\rangle=\left\langle\mu_{j}^{2}\right\rangle \exp \left(-t / \tau_{j}\right) \cos \left(2 \pi c \widetilde{\nu}_{j} t\right) .
$$

In this model we assume that the mode is homogeneously broadened. The line-shape function derived from Eq. (5) is expressed as 


$$
\begin{aligned}
I_{M M, j}(\widetilde{\nu})= & \frac{\left\langle\mu_{j}^{2}\right\rangle}{4 \pi^{2} c}\left[\frac{\frac{1}{\tau_{j}}}{\left(\widetilde{\nu}_{j}-\widetilde{\nu}\right)^{2}+\left(\frac{1}{2 \pi c \tau_{j}}\right)^{2}}\right. \\
& \left.+\frac{\frac{1}{\tau_{j}}}{\left(\widetilde{\nu}_{j}+\widetilde{\nu}\right)^{2}+\left(\frac{1}{2 \pi c \tau_{j}}\right)^{2}}\right] .
\end{aligned}
$$

Here $\left\langle\mu_{j}^{2}\right\rangle$ is the initial value of $\left\langle\mu_{j}(t) \mu_{j}(0)\right\rangle$, representing a magnitude of the dipole moment. It should be noted that the line-shape function of Eq. (6) is represented as a sum of the two terms. For mid-IR vibrational spectroscopy, the first term in Eq. (6) is generally included in the line-shape function, and the second term is eliminated under the assumption of the rotating wave approximation (RWA). In the $\mathrm{THz}$ region, however, the RWA cannot always be applied because a bandwidth of a mode is not necessarily much less than the oscillating frequency.

\section{Method of $a b$ initio Calculation}

Self-consistent reaction field (SCRF) $a b$ initio molecular orbital calculations on zwitterionic L,L-cystine, L-cysteine, and L-methionine were performed at the Hartree-Fock HF/6-31g(d), and HF/6-311++g(d,p) levels, respectively, using the Gaussian 03 version of the program. ${ }^{43}$ The Onsager dipole-sphere model ${ }^{44}$ was used in the SCRF calculations because it permits full structural optimizations and vibrational frequency calculations at a relatively low computational cost. X-ray crystallography ${ }^{45-47}$ revealed that amino acids in the crystalline state exist as one or more independent forms of the conformers as the zwitterion and the amino and thiol group hydrogen atoms participate in a threedimensional network of hydrogen bonds. The effect of the local environment has been taken into account for the calculations of zwitterions. Therefore in the present study the geometry optimizations were carried out by fixing the cavity radii in the presence of the reaction field in a nonpolar medium. We chose a dielectric constant $\epsilon$ of 4.88, which is equal to a dielectric constant of solid $\mathrm{KBr}$, by a somewhat arbitrary manner. The cavity radii of the L-cysteine and L,L-cystine were estimated by a selfconsistent field energy optimization. ${ }^{44}$ The cavity radii used for the SCRF calculations were chosen to be 5.20, 3.20 , and $3.26 \AA$ for L,L-cystine, L-cysteine, and L-methionine, ${ }^{36}$ respectively. The harmonic vibrational frequencies were calculated for the optimized geometries by analytic second differentiation of the energy with respect to nuclear displacements. To verify whether the lowfrequency spectra correspond to the neutral molecule, we have further optimized the geometry of selected conformers starting from the structure of the zwitterions in the absence of the dielectric medium with HF/6-311+ $+\mathrm{g}(\mathrm{d}, \mathrm{p})$.

\section{RESULTS AND DISCUSSION}

\section{A. Characterization of the Apparatus}

The frequency-dependent response efficiency of the EO sampling can be characterized by the coherence length function $l_{c}(\widetilde{\nu})^{48}$ and the frequency response function $G(\widetilde{\nu}){ }^{49}$ The coherence length function, based on the phase matching between the phase velocity of the $\mathrm{THz}$ wave and the group velocity of the optical pulse in the EO crystal, is expressed by ${ }^{48}$

$$
\begin{aligned}
l_{c}(\widetilde{\nu}) & =\frac{1}{2 \widetilde{\nu}\left|n_{g}\left(\lambda_{\text {opt }}\right)-n_{T H z}(\widetilde{\nu})\right|} \\
& =\frac{1}{2 \widetilde{\nu}\left|n_{\text {opt }}\left(\lambda_{\text {opt }}\right)-\lambda_{\text {opt }}\left(\frac{\mathrm{d} n_{\text {opt }}}{\mathrm{d} \lambda}\right)_{\lambda=\lambda_{\text {opt }}}-n_{T H z}(\widetilde{\nu})\right|}, \\
& n_{g}\left(\lambda_{\text {opt }}\right)=n_{\text {opt }}\left(\lambda_{\text {opt }}\right)-\lambda_{\text {opt }}\left(\frac{\mathrm{d} n_{\text {opt }}}{\mathrm{d} \lambda}\right)_{\lambda=\lambda_{\text {opt }}} .
\end{aligned}
$$

$n_{g}\left(\lambda_{\text {opt }}\right)$ is the effective refractive index for optical group velocity centered at $\lambda=\lambda_{\text {opt }}$. The subscripts $T H z$ and opt indicate parameters in the $\mathrm{THz}$ and optical regions, respectively. $n$ is the refractive index of ZnTe, and $\lambda$ is the wavelength. The coherence length function shown in Fig. 2(a) is calculated for the case of the EO sampling using a (110)ZnTe crystal probed by a femtosecond pulse centered at $800 \mathrm{~nm}$. Refractive indices $n_{T H z}(\widetilde{\nu})$ and $n_{\text {opt }}\left(\lambda_{\text {opt }}\right)$ are calculated by using the parameters listed in Ref. 48. At $80 \mathrm{~cm}^{-1}, l_{c}(\widetilde{\nu})$ provides $1 \mathrm{~mm}$ in coherence length, which is equal to the thickness of the (110)ZnTe crystal in our $\mathrm{THz}$ TDS system. The coherence length diverges around $65 \mathrm{~cm}^{-1}$ where the phase mismatch is zero. It decreases rapidly to zero in coherence length over $80 \mathrm{~cm}^{-1}$ because of the steep increase of $n_{T H z}(\widetilde{\nu})$, resulting from the transverse optical phonon resonance of $\mathrm{ZnTe}$ at $\sim 180 \mathrm{~cm}^{-1}$. The frequency response function is represented by ${ }^{49}$

$$
\begin{aligned}
G(\widetilde{\nu}) & =\left|\frac{t_{12}(\widetilde{\nu})}{\delta(\widetilde{\nu})} \int_{0}^{\delta(\widetilde{\nu})} \exp [i 2 \pi c \tilde{\nu} t] \mathrm{d} t\right| \\
& =t_{12}(\widetilde{\nu})\left|\frac{\exp [i 2 \pi c \tilde{\nu} \delta(\widetilde{\nu})]-1}{i 2 \pi c \tilde{\nu} \delta(\widetilde{\nu})}\right|, \\
\delta(\widetilde{\nu}) & =\frac{n_{g}\left(\lambda_{\text {opt }}\right)-n_{T H z}(\widetilde{\nu})}{c} d_{\mathrm{ZnTe}} .
\end{aligned}
$$

$t_{12}(\widetilde{\nu})$ is the real Fresnel transmission coefficient at the surface traveling from 1 (air) to 2 (ZnTe) with an incidence angle of $0^{\circ} . \delta(\widetilde{\nu})$ is the accumulated group-velocity mismatch time, and $d_{\mathrm{ZnTe}}$ is the thickness of the ZnTe crystal. The frequency response function is the time average of the $\mathrm{THz}$ electric field across the time interval of $\delta(\widetilde{\nu})$, representing the EO efficiency as $G(\widetilde{\nu})$ multiplied by 
$d_{\mathrm{ZnTe}}$. The frequency response function is represented by the solid curve in Fig. 2(b). Figure 2(b) shows $G(\widetilde{\nu})$ under the condition that the (110)ZnTe is $1 \mathrm{~mm}$ thick and the probe pulses are centered at $800 \mathrm{~nm}$. The dotted curve depicts the real Fresnel transmission, representing the upper bound of the EO efficiency. The frequency response below $65 \mathrm{~cm}^{-1}$ is nearly constant, and most of the EO efficiency is determined by the Fresnel transmission. On the other hand, it decreases steeply in the vicinity at $80 \mathrm{~cm}^{-1}$, reducing to zero at $90 \mathrm{~cm}^{-1}$. Figures 2(a) and 2(b) therefore indicate that the $\mathrm{THz}$ detection of our $\mathrm{THz}$ TDS system has constant efficiency up to $80 \mathrm{~cm}^{-1}$, and the upper limit of the detectable range is $90 \mathrm{~cm}^{-1}$ in the system.

Figure 3 shows the electric fields of the pulsed $\mathrm{THz}$ radiation in the time domain, which are generated from the (100)InAs surface under the external magnetic-flux density $\mathbf{B}$. The plus sign of $\mathbf{B}$ is defined as the direction from top to bottom. Figures 3(a)-3(c) show the $\mathrm{THz}$ radiation under $\mathbf{B}=0 \mathrm{~T}, \mathbf{B}=+1.6 \mathbf{T}$, and $\mathbf{B}=-1.6 \mathrm{~T}$, respectively, representing enhancement of the $\mathrm{THz}$ radiation induced by the external magnetic field. Figures 3(b) and 3(c), however, represent the polarity inversion with each other, suggesting that the ultrafast charge transports due to the photo-Dember effect at the InAs surface are modified by the Lorentz forces whose directions are dependent on the direction of $\mathbf{B}{ }^{50}$ Figure 4 represents the power spectra of the $\mathrm{THz}$ waves shown in Fig. 3. The total $\mathrm{THz}$ power increases by 5.8 times under $\mathbf{B}=+1.6 \mathbf{T}$ and by 10.1 times under $\mathbf{B}=-1.6 \mathrm{~T}$ compared with the power under $\mathbf{B}=0 \mathrm{~T}$. The enhancement obtained in our system is smaller than those reported in the earlier studies. ${ }^{51-53}$ The difference may come from the fact that the pump pulses are focused on the (100)InAs surface in $45^{\circ}$ incidence in our spectrometer, and therefore the $\mathrm{THz}$ wave under $\mathbf{B}=0$ includes the contribution of the $\mathrm{THz}$ radiation generating through the $\chi^{(2)}$ process. In this case smaller enhancement is expected

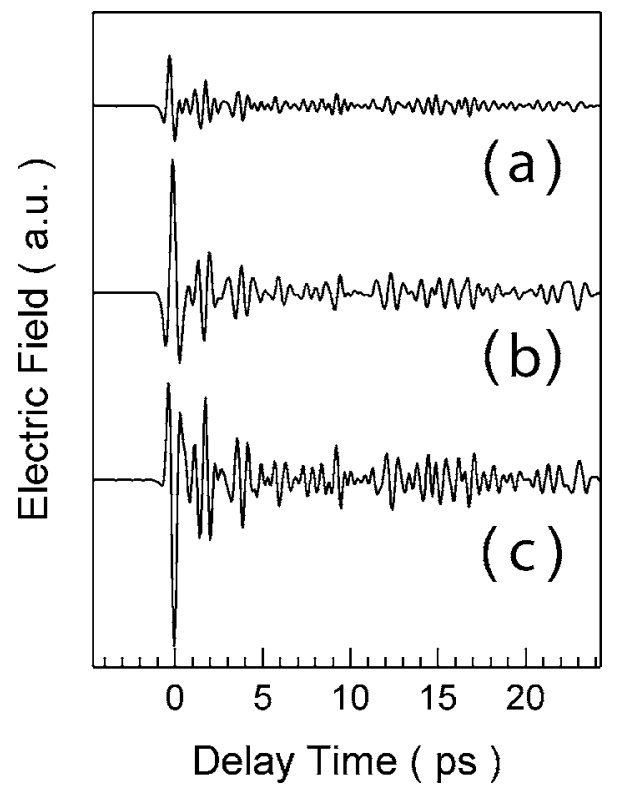

Fig. 3. THz waves generated from the (100)InAs and their dependence on magnetic fields: (a) $\mathbf{B}=0 \mathrm{~T}$, (b) $\mathbf{B}=+1.6 \mathrm{~T}$, (c) $\mathbf{B}=-1.6 \mathrm{~T}$.

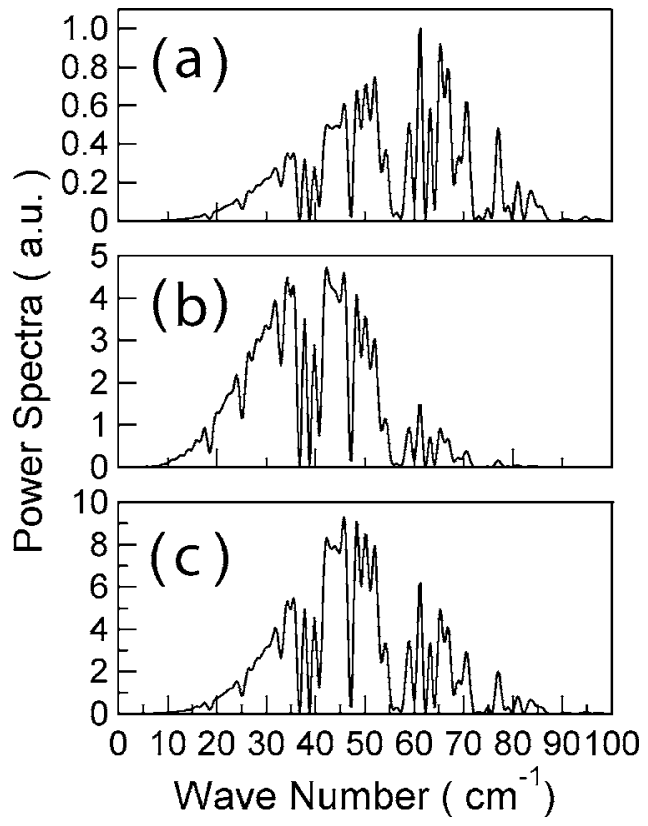

Fig. 4. Power spectra of THz radiation from the (100)InAs and their dependence on magnetic fields: (A) $\mathbf{B}=0 \mathrm{~T}$, (B) $\mathbf{B}=+1.6 \mathrm{~T}$, (C) $\mathbf{B}=-1.6 \mathrm{~T}$.

because a magnetic field has less influence on the $\chi^{(2)}$ process than that on the surface photocurrent. The larger enhancement in total power is obtained under $\mathbf{B}=-1.6 \mathrm{~T}$ rather than under $\mathbf{B}=+1.6 \mathrm{~T}$.

\section{B. Measurement and Analysis of Terahertz Spectra}

Figures 5(a)-5(d) show $\mathrm{THz}$ spectra of L,L-cystine, L-cysteine, L-methionine, and oxidized glutathione, respectively. The $\mathrm{THz}$ spectra of L,L-cystine show three sharp bands at 23.7, 49.7, and $68.9 \mathrm{~cm}^{-1}$ and those of L-cysteine show two broad bands at 45.1 and $54.7 \mathrm{~cm}^{-1}$. These spectra represent different features from each other, although the constituents of these biomolecules are similar where L,L-cystine is a dimer of L-cysteine with disulfide bonding. This result illustrates that $\mathrm{THz}$ spectra are sensitive to molecular structures even in the case of similar constituents. L-methionine represents much broader bands at 31.4 and $61.8 \mathrm{~cm}^{-1}$ in the $\mathrm{THz}$ spectra than L,L-cystine and L-cysteine, and oxidized glutathione shows featureless spectra. The peak frequencies as well as the bandwidths are different among the sulfur biomolecules investigated here. The molar absorption coefficient of each peak is summarized in Table 1. The spectral difference shown in Fig. 5 indicates that THz TDS can provide fingerprint $\mathrm{THz}$ spectra to identify the molecules.

These spectral features of the broadness are reflected in the parameter and $\tau_{j}$ in the damping harmonicoscillator model. In Fig. 6 the experimental data of L,Lcystine, L-cysteine, and L-methionine are recast into the complex permittivity spectra. The experimental results are shown by open circles. The simulated imaginary parts of the permittivity spectra by the damping oscillator model are expressed as the solid curves in the upper panels of Fig. 6. This model well reproduces the experimental spectra of the imaginary parts. Optimized parameters $\tilde{\nu}_{j},\left\langle\mu_{j}^{2}\right\rangle / V$, and $\tau_{j}$ are summarized in Table 1 . In the simu- 
lation one more band is needed to reproduce the highfrequency responses observed in Fig. 6. Parameters of these high-frequency bands are determined somehow arbitrarily. The real parts of the permittivity are calculated by the K-K transformation using the parameters listed in Table 1. They are shown by the solid curves in the lower panels of Fig. 6. The real parts of the permittivity are also reproduced well. Their baselines in the $\mathrm{THz}$ region cannot be determined only by $\mathrm{THz}$ TDS, and the calculated real parts are base shifted to obtain the best overlaps with the experimental spectra. It can be seen that the damping
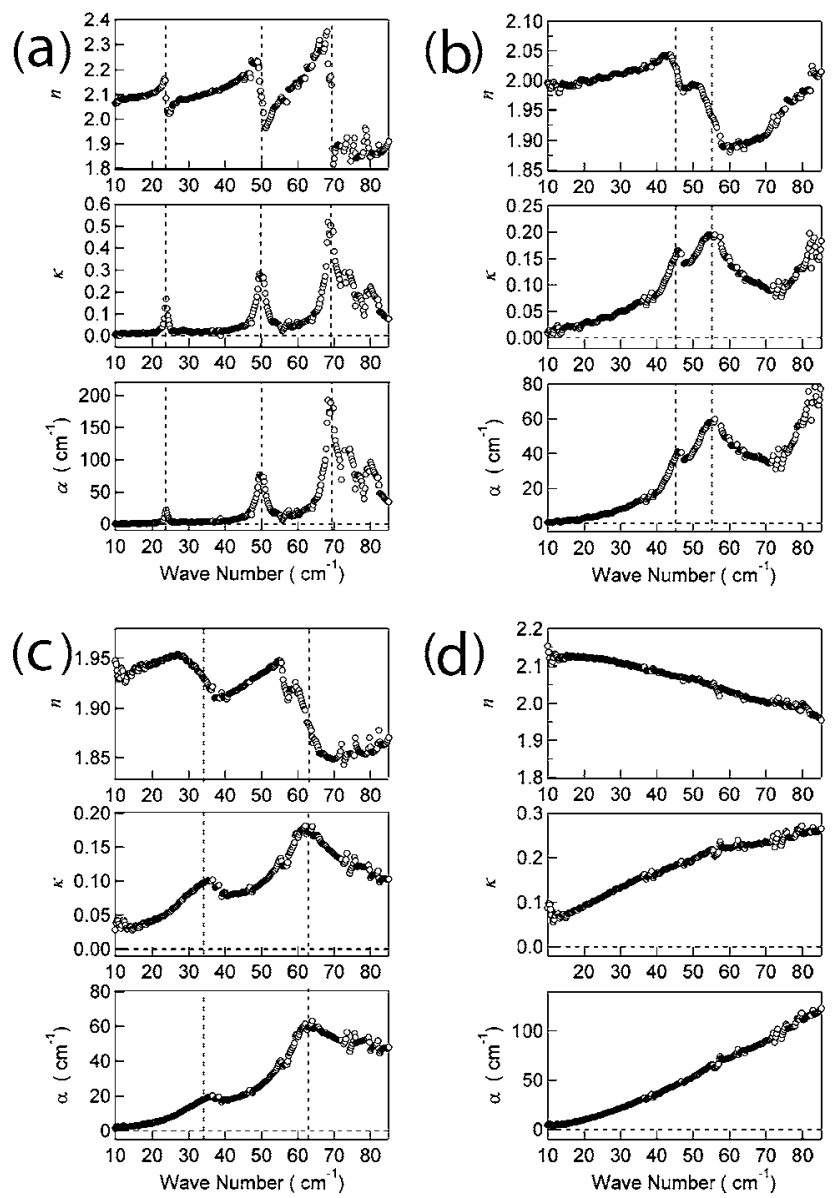

Fig. 5. THz spectra of sulfur biomolecules: (A) L,L-cystine, (B) L-cysteine, (C) L-methionine, (D) oxidized glutathione. Each spectral set shows refractive index, extinction coefficient, and absorption coefficient from top to bottom. harmonic-oscillator model well represents the permittivity spectra in the $\mathrm{THz}$ region in both the real and the imaginary parts.

The fifth column in Table 1 represents a square root of the initial value of the TCF of the $j$ th vibrational mode per one molecule, $\sqrt{\left\langle\mu_{j}^{2}\right\rangle}$. Let us discuss the physical meaning of this quantity. As we described before, there are generally a couple of mechanisms for the source of the damping time constant such as the reorientational relaxation of the dipole, pure dephasing, and lifetime of the excited vibrational state. If the reorientational relaxation is the main source for the damping, the quantity of $\sqrt{\left\langle\mu_{j}^{2}\right\rangle}$ represents the magnitude of the dipole. In the case where mechanisms of the pure dephasing or the lifetime broadening are dominant, the square root of the initial value corresponds to the amplitude of the oscillation of the dipole. In this work we rule out the possibility of the contribution of the reorientational relaxation because of the solid sample. Therefore we now assign the quantity of $\sqrt{\left\langle\mu_{j}^{2}\right\rangle}$ to represent the oscillation amplitude of the dipole. Since the oscillating amplitude is normally smaller than the magnitude of the dipole, this spectral analysis provides a rough estimation for the upper limit of the dipole magnitude. The values $\sqrt{\left\langle\mu_{j}^{2}\right\rangle}$ of the three modes observed for L,L-cystine are $0.27 \mathrm{D}$ at $23.7 \mathrm{~cm}^{-1}, 0.42 \mathrm{D}$ at $49.7 \mathrm{~cm}^{-1}$, and $0.55 \mathrm{D}$ at $68.9 \mathrm{~cm}^{-1}$. These values are comparable with static dipole moments of polar molecules (0.36 $\mathrm{D}$ for toluene, 1.01 $\mathrm{D}$ for chloroform, and 1.71 $\mathrm{D}$ for methanol). The low-frequency modes in L-cysteine and L-methionine also show similar values of $\sqrt{\left\langle\mu_{j}^{2}\right\rangle}$ to those in L,L-cystine.

Next let us discuss the relation between the oscillation magnitude and the absorption coefficient. The molar absorption coefficients of the bands are quite small compared to the band intensities with the dipole-allowed transition in the mid-IR region, although the oscillation magnitudes of the low-frequency bands obtained by the simulation are large. From relation (1) one can see that the product of the refractive index and absorption coefficient $[n(\widetilde{\nu}) \alpha(\widetilde{\nu})]$ is related to the Fourier transform of the TCF of the dipole moment. The product is proportional to a factor of $\widetilde{\nu}[1-\exp (-\beta h c \widetilde{\nu})]$, which is approximated to be $\beta h c \widetilde{\nu}^{2}$ for the case of $\beta h c \widetilde{\nu} \ll 1$, and $\widetilde{\nu}$ for the case of $\beta h c \widetilde{\nu}$ $\gg 1$. Therefore it is expected that the product of $n(\widetilde{\nu}) \alpha(\widetilde{\nu})$ is roughly proportional to $\widetilde{\nu}^{\alpha}$ where $\alpha$ varies from one to two, and consequently the absorption coefficient in the low-

Table 1. Absorption Coefficients and Optimized Parameters for the Sulfur-Containing Biomolecules by the Damping Harmonic-Oscillator Model

\begin{tabular}{|c|c|c|c|c|c|}
\hline Biomolecule & $\left(\mathrm{cm}^{-1}\right)$ & $\begin{array}{c}\left\langle\mu_{j}^{2}\right\rangle / V \\
\left(10^{-33} \mathrm{C}^{2} \mathrm{~m}^{-1}\right)\end{array}$ & $\begin{array}{c}\tau_{j} \\
(\mathrm{ps})\end{array}$ & $\begin{array}{c}\sqrt{\left\langle\mu_{j}^{2}\right\rangle} \\
\left(10^{-30} \mathrm{~cm}\right)\end{array}$ & $\begin{array}{c}\text { Molar Absorption } \\
\text { Coefficient } \\
\left(\mathrm{mol} \mathrm{l}^{-1} \mathrm{~cm}^{-1}\right)\end{array}$ \\
\hline & $23.74 \pm 0.04$ & $2.94 \pm 0.27$ & $10.6 \pm 1.3$ & $0.88 \pm 0.27$ & 3.4 \\
\hline \multirow[t]{2}{*}{ L,L-cystine } & $49.74 \pm 0.04$ & $7.24 \pm 0.28$ & $3.97 \pm 0.19$ & $1.38 \pm 0.27$ & 12.6 \\
\hline & $68.87 \pm 0.02$ & $12.90 \pm 0.29$ & $3.31 \pm 0.09$ & $1.85 \pm 0.28$ & 34.3 \\
\hline \multirow[t]{2}{*}{ L-cysteine } & $45.05 \pm 0.13$ & $7.04 \pm 0.41$ & $1.27 \pm 0.07$ & $1.02 \pm 0.25$ & 3.7 \\
\hline & $54.67 \pm 0.12$ & $10.28 \pm 0.44$ & $1.05 \pm 0.04$ & $1.24 \pm 0.26$ & 5.2 \\
\hline \multirow[t]{2}{*}{ L-methionine } & $31.41 \pm 0.27$ & $11.26 \pm 1.19$ & $0.59 \pm 0.04$ & $1.56 \pm 0.48$ & 2.3 \\
\hline & $61.77 \pm 0.12$ & $11.60 \pm 0.76$ & $0.70 \pm 0.03$ & $1.50 \pm 0.38$ & 7.0 \\
\hline
\end{tabular}



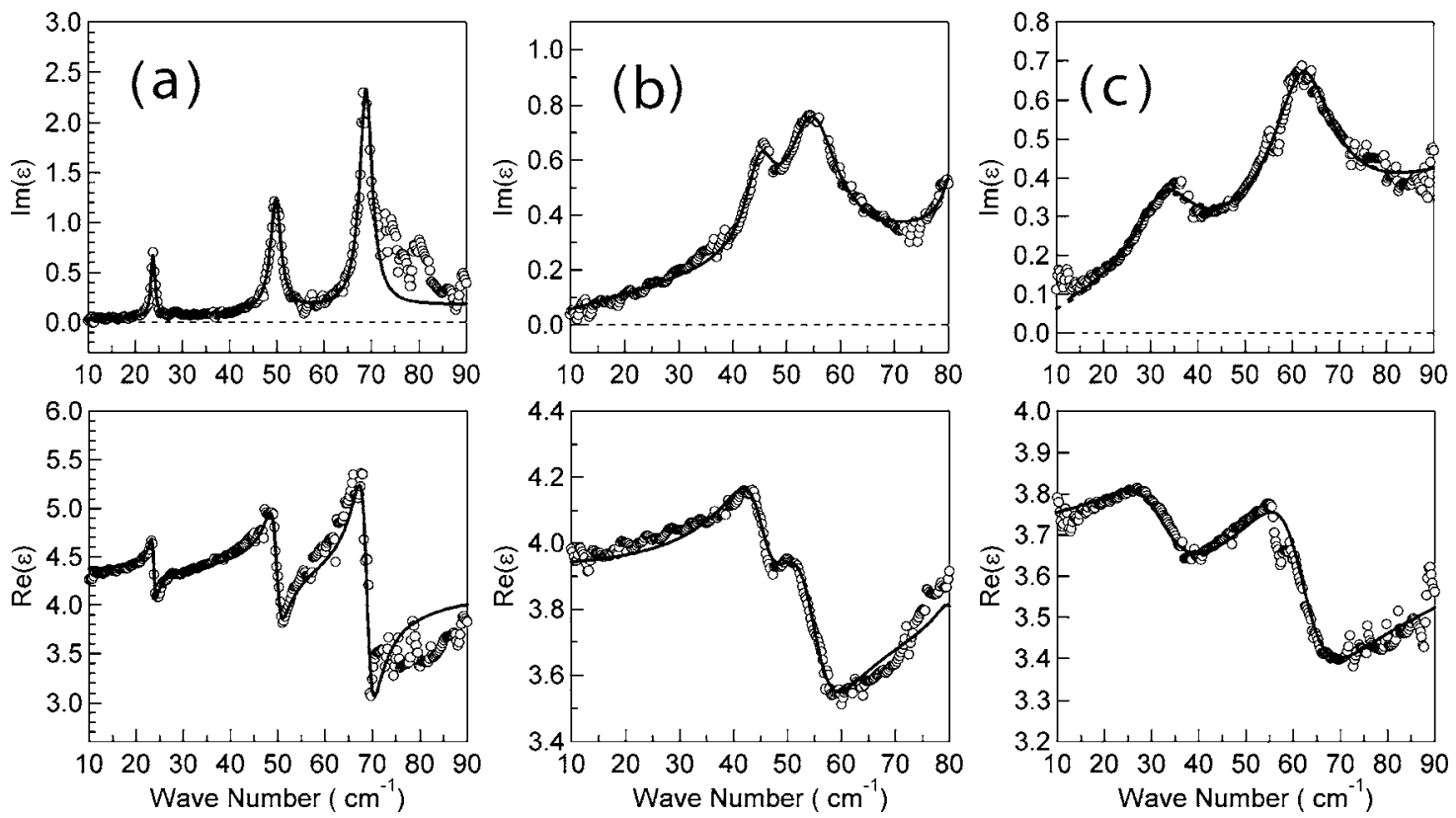

Fig. 6. Complex permittivity spectra of sulfur biomolecules: (a) L,L-cystine, (b) L-cysteine, (c) L-methionine. Upper panels show imaginary parts and lower panels show real parts. Open circles are experimental permittivities measured by THz TDS. Simulated spectra in the imaginary parts and calculated spectra in the real parts are represented by solid curves.

Table 2. Calculated Harmonic Vibrational Frequencies and IR Intensities of Zwitterionic L,L-Cysteine, L-Cysteine, and L-Methionine

\begin{tabular}{|c|c|c|c|c|c|}
\hline & \multicolumn{2}{|c|}{ Gas Phase } & \multicolumn{2}{|c|}{ Nonpolar Dielectric Medium $(\epsilon=4.88)$} & \multirow[b]{2}{*}{ Approximate Assignments } \\
\hline & $\begin{array}{l}\text { Calculated } \\
\text { Frequency } \\
\left(\mathrm{cm}^{-1}\right)\end{array}$ & $\begin{array}{c}I \mathrm{IR} \\
(\mathrm{km} / \mathrm{mol})\end{array}$ & $\begin{array}{l}\text { Calculated } \\
\text { Frequency }\left(\mathrm{cm}^{-1}\right)\end{array}$ & $I_{\mathrm{IR}}(\mathrm{km} / \mathrm{mol})$ & \\
\hline \multirow[t]{4}{*}{ L,L-cystine ${ }^{a}$} & 28.12 & 20.72 & 18.79 & 50.45 & $\begin{array}{l}\text { Relative } \mathrm{C}^{\alpha} \mathrm{H}\left(\mathrm{NH}_{3}\right) / \mathrm{C}^{\alpha^{\prime}} \mathrm{H}\left(\mathrm{NH}_{3}\right) \text { deformation } \\
\quad \text { (relative motion of the two cysteins) }\end{array}$ \\
\hline & 34.78 & 10.69 & 36.40 & 13.41 & Relative $\mathrm{C}^{\beta}-\mathrm{C}^{\alpha}\left(\mathrm{NH}_{3}\right) / \mathrm{C}^{\beta^{\prime}}-\mathrm{C}^{\alpha^{\prime}}\left(\mathrm{NH}_{3}\right)$ torsion \\
\hline & 66.78 & 17.59 & 63.23 & 20.62 & $\begin{array}{c}\text { Relative } \mathrm{C}^{\beta} \mathrm{H}_{2}-\mathrm{C}^{\alpha}\left(\mathrm{NH}_{3}\right) / \mathrm{C}^{\beta^{\prime}} \mathrm{H}_{2}-\mathrm{C}^{\alpha^{\prime}}\left(\mathrm{NH}_{3}\right) \\
\text { deformation (relative motion of the two cystines) }\end{array}$ \\
\hline & 72.05 & 36.97 & 64.32 & 37.24 & Relative $\mathrm{C}-\mathrm{C}^{\alpha} / \mathrm{C}-\mathrm{C}^{\alpha^{\prime}}$ torsion $+\mathrm{NH}_{3}$ torsion \\
\hline \multirow[t]{2}{*}{ L-cysteine $^{a}$} & 61.90 & 3.85 & 75.59 & 5.74 & $\mathrm{COO}$ torsion \\
\hline & 78.17 & 9.27 & 90.61 & 34.20 & $\mathrm{C}^{\beta}-\mathrm{C}^{\alpha}$ torsion \\
\hline \multirow[t]{3}{*}{ L-methionine ${ }^{b}$} & 38.54 & 2.83 & 44.77 & 36.43 & $\mathrm{C}^{\alpha}-\mathrm{C}^{\beta}$ torsion \\
\hline & 52.23 & 1.69 & 54.62 & 9.79 & COO torsion \\
\hline & 57.03 & 1.06 & 69.92 & 5.27 & $\mathrm{~S}-\mathrm{CH}_{3}$ deformation \\
\hline
\end{tabular}

${ }^{a}$ The calculations were performed at the HF/6-31g(d) level of theory for both gas phase and a nonpolar dielectric medium.

${ }^{b}$ The calculations were performed at the HF/6-311++g(d,p) level of theory for both gas phase and a nonpolar dielectric medium.

frequency region is quite small compared with those in the high-frequency region; even the magnitudes of the oscillation of the bands are similar. The method of $\mathrm{THz}$ analysis presented here allows us to estimate the upper limit of the magnitude of the dipoles in the low-frequency region from the data taken by $\mathrm{THz}$ TDS, which can serve as a benchmark for theoretical calculation.

\section{Results of $a b$ initio Calculation}

Normal modes of low-frequency vibrations for the zwitterionic L,L-cystine and L-cysteine in the gas phase and in the nonpolar medium were calculated at the $\mathrm{HF} / 6$ $-31 \mathrm{~g}(\mathrm{~d})$ levels of theory. Frequency calculations for the zwitterionic and neutral L-methionine in the gas phase and in the nonpolar medium were calculated at the $\mathrm{HF} / 6-311++\mathrm{g}(\mathrm{d}, \mathrm{p})$ levels of theory. The calculated frequencies, scaled down by a factor 0.9061, together with their assignments are enumerated in Table 2. For L,Lcystine we have found that one zwitterionic-zwitterionic type conformer gives results that qualitatively agree with our observation. However, the agreement is poor in a quantitative level, and further improvement is necessary to assign the vibrational modes. The optimized structures of the L,L-cystine, L-cysteine, and L-methionine obtained by SCRF calculation are presented in Fig. 7. In the case of the L-cysteine molecule, we have chosen two zwitterionic 


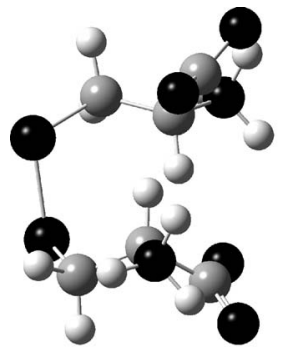

(a)

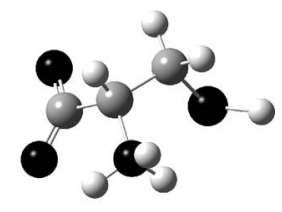

(b)

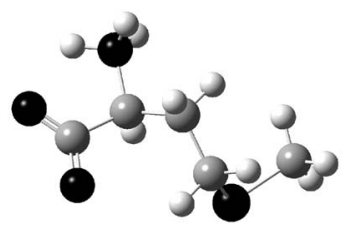

(c)

Fig. 7. Molecular structures optimized by SCRF $a b$ initio molecular orbital calculation of (a) L,L-cystine, (b) L-cysteine, and (c) L-methionine at the HF/6-31g(d), HF/6-31g(d), and HF/6 $-311++g(d, p)$, levels, respectively. The symbols $\mathrm{C}^{\alpha}$ and $\mathrm{C}^{\beta}$ are used in Table 2.

L-cysteine (A) and L-cysteine (B) conformers revealed by x-ray crystallography. ${ }^{47}$ The results of the L-cysteine (A) conformer are shown in Table 2. Although we have verified that this geometry predicts the frequencies that reasonably agree well with other experimentally observed high-frequency modes ${ }^{40}$ the calculated frequencies are higher than the frequencies observed in this work (Table 1). In the case of L-methionine zwitterions, the harmonic vibrational frequencies for the optimized geometry of the tGG conformer gives closer results with our observation in a qualitative level. ${ }^{36}$ In the crystalline state, L-methionine exists as methionine $\mathrm{A}$ and methionine $\mathrm{B}^{46,47}$ There is a reasonable agreement between the tGG and methionine B structures. It can be concluded that the agreement between the calculated results and the experimental observation is not satisfactory in a quantitative level. The presence of a three-dimensional network of hydrogen bonds may affect the molecular structures and the vibrational frequencies of the normal modes, especially for the low-frequency modes. Furthermore, the observed bands may be due to the intermolecular hydrogen bonds. Fischer et al. observed $\mathrm{THz}$ spectra of thymine in the crystalline state at $10 \mathrm{~K}$, and they performed discrete density functional theory calculation by taking into account the spatial arrangement of the molecules in the crystalline structure. ${ }^{54}$ They concluded that four of the observed bands arise from intermolecular motion of the hydrogen bond system. For the present case, it will be interesting to perform such a calculation by considering the crystalline structure to investigate vibrational modes of the hydrogen bond network.

\section{SUMMARY}

The magnetic field (=1.6 T) applied to the (100)InAs enhances the $\mathrm{THz}$ radiation from its surface as well as modifies the frequency components of the generated $\mathrm{THz}$ radiation. The enhancement factor is dependent on the direction of the magnetic field. The frequency region of the EO sampling by use of the (110)ZnTe in $1 \mathrm{~mm}$ thickness is discussed by the coherence length and the frequency response function. The three sulfur biomolecules investigated, L,L-cystine, L-cysteine, and L-methionine, show characteristic spectral features, and THz TDS may provide direct fingerprints of the molecule, whereas oxidized glutathione shows featureless spectra. The $\mathrm{THz}$ spectra of L-cysteine and L,L-cystine significantly differ in their peak frequencies and bandwidths in spite of their similar constituents. We present a method to analyze the real and imaginary parts of the $\mathrm{THz}$ permittivity and obtain the magnitude of the oscillation of the dipole in the low-frequency modes. We conclude that our spectral analysis of THz TDS based on the theoretical model is a tool to investigate the dipole dynamics in the lowfrequency region in the condensed phases. We have also performed $a b$ initio calculations for L,L-cystine, L-cysteine, and L-methionine. We obtained some reasonable agreements between the experiment and calculations, but overall the agreement is rather poor on a quantitative level, suggesting the importance of the intermolecular interactions such as hydrogen bond.

\section{ACKNOWLEDGMENTS}

We acknowledge Hideyuki Ohtake, Nobuhiko Sarukura, Masanori Hangyo, Kiyomi Sakai, Masahiko Tani, Kaoru Ohta, and Koichirou Tanaka for variable discussion and helpful comments. We also thank Gad Fischer for helpful discussions. This work was partially supported by a Japan Society for the Promotion of Science research grant for the Future Program.

$\mathrm{K}$. Yamamoto is now with the Institute of Laser Engineering, Osaka University, 2-1 Yamadaoka, Suita, Osaka 565-0871, Japan.

\section{REFERENCES}

1. D. H. Auston, K. P. Cheung, and P. R. Smith, "Picosecond photoconducting Hertzian dipoles," Appl. Phys. Lett. 45, 284-286 (1984).

2. M. van Exter, C. Fattinger, and D. Grischkowsky, "Highbrightness terahertz beams characterized with an ultrafast detector," Appl. Phys. Lett. 55, 337-339 (1989).

3. S. L. Chuang, S. S. Rink, B. I. Greene, P. N. Saeta, and A. F. J. Levi, "Optical rectification at semiconductor surfaces," Phys. Rev. Lett. 68, 102-105 (1992).

4. A. Rice, Y. Jin, X. F. Ma, X.-C. Zhang, D. Bliss, J. Larkin, and M. Alexander, "Terahertz optical rectification from $<110>$ zinc-blend crystals," Appl. Phys. Lett. 64, 1324-1326 (1994).

5. Q. Wu and X.-C. Zhang, "Free-space electro-optic sampling of terahertz beams," Appl. Phys. Lett. 67, 3523-3525 (1995).

6. X.-C. Zhang, B. B. Hu, J. T. Darrow, and D. H. Auston, "Generation of femtosecond electromagnetic pulses from 
semiconductor surfaces,” Appl. Phys. Lett. 56, 1011-1013 (1990).

7. X.-C. Zhang and D. H. Auston, "Optoelectronic measurement of semiconductor surfaces and interfaces with femtosecond optics," J. Appl. Phys. 71, 326-338 (1992).

8. J. E. Pedersen and S. R. Keiding, "THz time-domain spectroscopy of nonpolar liquids," IEEE J. Quantum Electron. 28, 2518-2522 (1992).

9. L. Thrane, R. H. Jacobsen, P. U. Jepsen, and S. R. Keiding, "THz reflection spectroscopy of liquid water," Chem. Phys. Lett. 240, 330-333 (1995).

10. S. R. Keiding, "Dipole correlation functions in liquid benzenes measured with terahertz time domain spectroscopy,” J. Phys. Chem. A 101, 5250-5254 (1997).

11. C. Rønne, L. Thrane, P.-O. Åstrand, A. Wallqvist, K. V. Mikkelsen, and S. R. Keiding, "Investigation of the temperature dependence of dielectric relaxation in liquid water by $\mathrm{THz}$ reflection spectroscopy and molecular dynamics simulation,” J. Chem. Phys. 107, 5319-5331 (1997)

12. C. Rønne, P.-O. Åstrand, and S. R. Keiding, "THz spectroscopy of liquid $\mathrm{H}_{2} \mathrm{O}$ and $\mathrm{D}_{2} \mathrm{O}$," Phys. Rev. Lett. 82, 2888-2891 (1999).

13. C. Rønne, K. Jensby, B. J. Loughnane, J. Fourkas, O. F. Nielsen, and S. R. Keiding, "Temperature dependence of the dielectric function of $\mathrm{C}_{6} \mathrm{H}_{6}(1)$ and $\mathrm{C}_{6} \mathrm{H}_{5} \mathrm{CH}_{3}(1)$ measured with $\mathrm{THz}$ spectroscopy," J. Chem. Phys. 113, 3749-3756 (2000)

14. J. T. Kindt and C. A. Schmuttenmaer, "Far-infrared dielectric properties of polar liquids probed by femtosecond terahertz pulse spectroscopy," J. Phys. Chem. 100, 10373-10379 (1996).

15. D. S. Venables and C. A. Schmuttenmaer, "Far-infrared spectra and associated dynamics in acetonitrile-water mixtures measured with femtosecond $\mathrm{THz}$ pulse spectroscopy," J. Chem. Phys. 108, 4935-4944 (1998).

16. D. S. Venables, A. Chiu, and C. A. Schmuttenmaer, "Structure and dynamics of nonaqueous mixtures of dipolar liquids. I. Infrared and far-infrared spectroscopy," J. Chem. Phys. 113, 3243-3248 (2000).

17. D. S. Venables and C. A. Schmuttenmaer, "Spectroscopy and dynamics of mixtures of water with acetone, acetonitrile, and methanol," J. Chem. Phys. 113, 11222-11236 (2000).

18. M. C. Beard, G. M. Turner, and C. A. Schmuttenmaer, "Terahertz spectroscopy," J. Phys. Chem. B 106, 7146-7159 (2002).

19. M. L. T. Asaki, A. Redondo, T. A. Zawodzinski, and A. J. Taylor, "Dielectric relaxation of electrolyte solutions using terahertz transmission spectroscopy," J. Chem. Phys. 116, 8469-8482 (2002).

20. M. L. T. Asaki, A. Redondo, T. A. Zawodzinski, and A. J. Taylor, "Dielectric relaxation and underlying dynamics of acetonitrile and 1-ethyl-3-methylimidazolium triflate mixtures using $\mathrm{THz}$ transmission spectroscopy," J. Chem. Phys. 116, 10377-10385 (2002).

21. A. G. Markelz, A. Roitberg, and E. J. Heilweil, "Pulsed terahertz spectroscopy of DNA, bovine serum albumin and collagen between 0.1 and $2.0 \mathrm{THz}$," Chem. Phys. Lett. 320, 42-48 (2000).

22. M. Brucherseifer, M. Nagel, P. H. Bolivar, H. Kurz, A. Bosserhoff, and R. Büttner, "Label-free probing of the binding state of DNA by time-domain terahertz sensing," Appl. Phys. Lett. 77, 4049-4051 (2000).

23. K. Yamamoto, K. Tominaga, H. Sasakawa, A. Tamura, H. Murakami, H. Ohtake, and N. Sarukura, "Far-infrared absorption measurements of polypeptides and cytochrome $c$ by $\mathrm{THz}$ radiation," Bull. Chem. Soc. Jpn. 75, 1083-1092 (2002).

24. M. Walther, B. Fischer, M. Schall, H. Helm, and P. U. Jepsen, "Far-infrared vibrational spectra of all-trans, 9-cis and 13-cis retinal measured by $\mathrm{THz}$ time-domain spectroscopy," Chem. Phys. Lett. 332, 389-395 (2000).

25. M. Walther, P. Plochocka, B. Fischer, H. Helm, and P. U.
Jepsen, "Collective vibrational modes in biological molecules investigated by terahertz time-domain spectroscopy," Biopolymers 67, 310-313 (2002).

26. B. Yu, F. Zeng, Y. Yang, Q. Xing, A. Chechin, X. Xin, I. Zeylikovich, and R. R. Alfano, "Torsional vibrational modes of tryptophan studied by terahertz time-domain spectroscopy,” Biophys. J. 86, 1649-1654 (2004).

27. P. F. Taday, I. V. Bradley, and D. D. Arone, "Terahertz pulse spectroscopy of biological materials: L-glutamic acid," J. Biol. Phys. 29, 109-115 (2003).

28. M. R. Kutteruf, C. M. Brown, L. K. Iwaki, M. B. Campbell, T. M. Korter, and E. J. Heilweil, "Terahertz spectroscopy of short-chain polypeptides," Chem. Phys. Lett. 375, 337-343 (2003).

29. K. Saitow, H. Ohtake, N. Sarukura, and K. Nishikawa, "Terahertz absorption spectra of supercritical $\mathrm{CHF}_{3}$ to investigate local structure through rotational and hindered rotational motions," Chem. Phys. Lett. 341, 86-92 (2001).

30. D. M. Mittleman, M. C. Nuss, and V. L. Colvin, "Terahertz spectroscopy of water in inverse micelles," Chem. Phys. Lett. 275, 332-338 (1997).

31. J. E. Boyd, A. Briskman, V. L. Colvin, and D. M. Mittleman, "Direct observation of terahertz surface modes in nanometer-sized liquid water pools," Phys. Rev. Lett. 87, 147401 (2001).

32. J. E. Boyd, A. Briskman, C. M. Sayes, D. M. Mittleman, and V. L. Colvin, "Terahertz vibrational modes of inverse micelles," J. Phys. Chem. B 106, 6346-6353 (2002).

33. R. G. Gordon, Correlation Functions for Molecular Motion, Vol. 3 of Advances in Magnetic Resonance (Academic, 1968), pp. 1-42.

34. B. J. Berne, "Time-dependent properties of condensed media," in Physical Chemistry, An Advanced Treatise, D. Henderson and H. Eyring, eds. (Academic, 1971), pp. $539-716$.

35. D. A. McQuarrie, Statistical Mechanics (University Science, 1976)

36. X. Cao and G. Fischer, "Conformational and infrared spectral studies of L-methionine and its N-deuterated isotopomer as isolated zwitterions," J. Phys. Chem. A 106 41-50 (2002).

37. A. Sawicka, P. Skurski, and J. Simons, "Excess electron attachment to disulfide-bridged L,L-cystine. An ab initio study," J. Phys. Chem. A 108, 4261-4268 (2004).

38. S. Gronert and R. A. J. O'Hair, "Ab initio studies of amino acid conformations. 1. The conformers of alanine, serine, and cysteine," J. Am. Chem. Soc. 117, 2071-2081 (1995).

39. K. Frimand, H. Bohr, K. J. Jalkanen, and S. Suhai, "Structures, vibrational absorption and vibrational circular dichroism spectra of L-alanine in aqueous solution: a density functional theory and RHF study," Chem. Phys. 255, 165-194 (2000).

40. P. Tarakeshwar and S. Manogaran, "Vibrational frequencies of cysteine and serine zwitterions-an $a b$ initio assignment," Spectrochim. Acta, Part A 51, 925-928 (1995).

41. K. Yamamoto, M. Yamaguchi, F. Miyamaru, M. Tani, M. Hangyo, T. Ikeda, A. Matsushita, K. Koide, M. Tatsuno, and Y. Minami, "Noninvasive inspection of C-4 explosive in mails by terahertz time-domain spectroscopy," Jpn. J. Appl. Phys., Part 2 43, L414-L417 (2004).

42. J. D. Jackson, Classical Electrodynamics, 3rd ed. (Wiley, 1998).

43. M. J. Frisch, G. W. Trucks, H. B. Schlegel, G. E. Scuseria, M. A. Robb, J. R. Cheeseman, J. A. Montgomery, Jr., T. Vreven, K. N. Kudin, J. C. Burant, J. M. Millam, S. S. Iyengar, J. Tomasi, V. Barone, B. Mennucci, M. Cossi, G. Scalmani, N. Rega, G. A. Petersson, H. Nakatsuji, M. Hada, M. Ehara, K. Toyota, R. Fukuda, J. Hasegawa, M. Ishida, T. Nakajima, Y. Honda, O. Kitao, H. Nakai, M. Klene, X. Li, J. E. Knox, H. P. Hratchian, J. B. Cross, C. Adamo, J. Jaramillo, R. Gomperts, R. E. Stratmann, O Yazyev, A. J. Austin, R. Cammi, C. Pomelli, J. W. Ochterski, P. Y. Ayala, K. Morokuma, G. A. Voth, P. Salvador, J. J. Dannenberg, V. G. Zakrzewski, S. Dapprich, A. D. Daniels, M. C. Strain, O. Farkas, D. K. Malick, A. D 
Rabuck, K. Raghavachari, J. B. Foresman, J. V. Ortiz, Q. Cui, A. G. Baboul, S. Clifford, J. Cioslowski, B. B. Stefanov, G. Liu, A. Liashenko, P. Piskorz, I. Komaromi, R. L. Martin, D. J. Fox, T. Keith, M. A. Al-Laham, C. Y. Peng, A. Nanayakkara, M. Challacombe, P. M. W. Gill, B. Johnson, W. Chen, M. W. Wong, C. Gonzalez, and J. A. Pople, Gaussian 03, Revision B.4 (Gaussian, Inc., 2003).

44. J. B. Foresman and A. Frisch, Exploring Chemistry with Electronic Structure Methods, 2nd ed. (Gaussian, Inc., 1996), p. 237.

45. K. A. Kerr, J. P. Ashmore, and T. F. Koetzle, "A neutron diffraction study of L-cysteine," Acta Crystallogr. 31, 2022-2026 (1975).

46. K. Torii and Y. Iitaka, "Crystal structures and molecular conformations of L-methionine and L-norleucine," Acta Crystallogr. 29, 2799-2807 (1973).

47. B. Dalhus and C. H. Görbitz, "Crystal structures of hydrophobic amino acids. I. Redeterminations of Lmethionine and L-valine at $120 \mathrm{~K}$," Acta Chem. Scand. 50, 544-548 (1996).

48. A. Nahata, A. S. Weling, and T. F. Heinz, "A wideband coherent terahertz spectroscopy system using optical rectification and electro-optic sampling," Appl. Phys. Lett. 69, 2321-2323 (1996).
49. Q. Wu and X.-C. Zhang, "7 terahertz broadband gap electro-optic sensor," Appl. Phys. Lett. 70, 1784-1786 (1997).

50. M. B. Johnston, D. M. Whittaker, A. Corchia, A. G. Davies, and E. H. Linfield, "Simulation of terahertz generation at semiconductor surfaces," Phys. Rev. B 65, 165301 (2002).

51. J. N. Heyman, P. Neocleous, D. Hebert, P. A. Crowell, T. Mueller, and K. Unterrainer, "Terahertz emission from GaAs and InAs in a magnetic field," Phys. Rev. B 64, 85202 (2001).

52. H. Ohtake, S. Ono, M. Sakai, Z. Liu, T. Tsukamoto, and N. Sarukura, "Saturation of THz-radiation power from femtosecond-laser-irradiated InAs in a high magnetic field," Appl. Phys. Lett. 76, 1398-1400 (2000).

53. N. Sarukura, H. Ohtake, S. Izumida, and Z. Liu, "High average-power $\mathrm{THz}$ radiation from femtosecond laserirradiated InAs in a magnetic field and its elliptical polarization characteristics," J. Appl. Phys. 84, 654-656 (1998).

54. B. M. Fischer, M. Walther, and P. Uhd Jepsen, "Farinfrared vibrational modes of DNA components studied by terahertz time-domain spectroscopy," Phys. Med. Biol. 47, 3807-3814 (2002). 